\title{
Efecto de alimentación húmeda de cerdos en finalización sobre el comportamiento productivo, composición de la canal y calidad de la carne
}

Néstor Arce Vázquez ${ }^{\mathrm{a}}$

Hugo Bernal Barragán ${ }^{\text {a* }}$

Nydia Corina Vásquez Aguilar ${ }^{a}$

Estela Garza Brenner ${ }^{a}$

Fernando Sánchez Dávila ${ }^{\text {a }}$

Adriana Morales Trejo ${ }^{b}$

Miguel Cervantes Ramírez ${ }^{b}$

${ }^{\text {a } U n i v e r s i d a d ~ A u t o ́ n o m a ~ d e ~ N u e v o ~ L e o ́ n . ~ F a c u l t a d ~ d e ~ A g r o n o m i ́ a . ~ C a m p u s ~ d e ~ C i e n c i a s ~}$ Agropecuarias UANL. Calle Francisco Villa S/N. Fracc. Ex-Hacienda "El Canadá". 66054 Gral. Escobedo, N.L., México.

${ }^{\text {b }}$ Universidad Autónoma de Baja California. Instituto de Ciencia Agrícolas. Mexicali. Baja California. México.

*Autor de correspondencia: hugo.bernalbr@uanl.edu.mx

\section{Resumen:}

El objetivo del estudio fue evaluar el efecto de la alimentación húmeda de cerdos en finalización (alimento:agua, 1:1) de una dieta basada en sorgo y harina de soya (15.0\% PC, $3,200 \mathrm{kcal} \mathrm{EM} / \mathrm{kg} \mathrm{MS}$ ), sobre el comportamiento productivo, composición de la canal y calidad de la carne. Dieciséis cerdos cruzados (York-Landrace x Duroc) de 68.4 $\pm 2.4 \mathrm{~kg}$ de peso fueron alojados individualmente, y asignados a dos tratamientos ( $\mathrm{n}=8$ repeticiones por tratamiento): AS, alimentación seca; AH, alimentación húmeda. Alimento fue ofrecido 
diariamente en dos porciones iguales ( 0800 y $1500 \mathrm{~h}$ ) durante cinco semanas. Semanalmente se registraron el peso vivo (PV) y el consumo de alimento individuales, para calcular ganancia diaria de peso (GDP) y eficiencia alimenticia (EA). Se midió la composición de la canal; la calidad de la carne fue medida en muestras de Longissimus dorsi. Cerdos AH tuvieron mayor $(P<0.05) \mathrm{PV}$ final (108.4 vs $101.9 \mathrm{~kg})$ y GDP $(1.043$ vs $0.990 \mathrm{~kg} /$ día) que los cerdos AS. Cerdos AS tuvieron menor consumo (semana 5) y EA (semana 3 ) que los cerdos AH (Interacción Tratamiento x Semana, $P<0.05$ ). Cerdos AH tuvieron pesos mayores de pierna, canal caliente y fría $(P<0.05)$. Lomo, costillas, paleta, contenido de proteína, capacidad de retención de agua y $\mathrm{pH}$ de la carne fueron similares $(P>0.05)$ entre tratamientos. Valores de dureza, adhesividad, masticabilidad y resistencia fueron menores $(P<0.05)$ en carne de cerdos $\mathrm{AH}$. En conclusión, los cerdos que recibieron alimento húmedo tuvieron mejor comportamiento productivo, composición de la canal y características de la carne que cerdos que recibieron alimento seco.

Palabras clave: Alimentación húmeda, Alimentación seca, Cerdos en finalización, Medición de canal.

Recibido: 19/12/2019

Aceptado: 02/11/2020

\section{Introducción}

El correcto manejo de la alimentación es importante para mejorar el bienestar animal, la eficiencia de crecimiento y los datos productivos de cerdos. Una posibilidad de mejorar los sistemas de alimentación para cerdos, consiste en mezclar alimento seco con agua, (proporciones entre 1: 1.0 y 1.5) $)^{(1)}$. Alimentación húmeda ha demostrado reducir el estrés en la transición de dieta líquida a sólida de lechones destetados ${ }^{(1,2,3)}$ y esto puede tener efectos beneficiosos tales como la reducción del uso de antibióticos en los sistemas de producción actuales $^{(4,5)}$. Además, la alimentación húmeda, mejora el consumo de agua y alimento ${ }^{(3,6)}, \mathrm{y}$ el suministro de nutrientes en cerdos crecimiento-finalización ${ }^{(7)}$, comparada con la alimentación seca, pudiendo esto favorecer el comportamiento productivo ${ }^{(4)} \sin$ afectar el contenido de grasa y calidad de la canal en $\operatorname{cerdos}^{(1,5)}$.

Características sensoriales, como la terneza, color y marmoleo, son importantes para determinar la calidad y la aprobación por el consumidor de la carne de $\operatorname{res}^{(8)}$, pollo ${ }^{(9)}$,

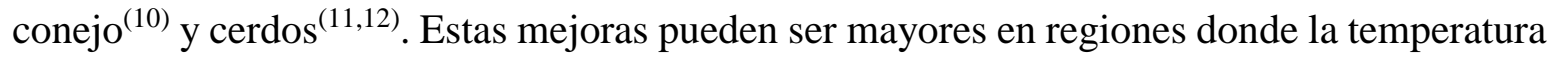
ambiente rebasa la zona termoneutral (confort) de los cerdos. Sin embargo, la información 
sobre el efecto de la alimentación húmeda es escasa para los sistemas de producción intensiva de carne de cerdo en zonas cálidas como el norte de México. Por lo tanto, el objetivo del presente estudio fue determinar el efecto de la alimentación húmeda a base de sorgo y pasta soya, sobre la tasa y eficiencia de crecimiento, comportamiento productivo, composición de la canal y calidad de la carne de los cerdos en finalización. La hipótesis del presente estudio fue que el consumo y utilización del alimento de los cerdos en estas condiciones climáticas podría mejorarse con la alimentación húmeda.

\section{Material y métodos}

Los cerdos utilizados, en la presente investigación, fueron atendidos de acuerdo con las pautas establecidas en la Norma Oficial Mexicana para el Cuidado de Animales ${ }^{(13)}$. El estudio se realizó en la Estación Experimental Porcina de la Unidad Académica Marín de la Facultad de Agronomía de la Universidad Autónoma de Nuevo León, ubicada en Marín, N.L., México. Se utilizaron 16 cerdos ( 8 hembras y 8 machos castrados; unidades experimentales) de la cruza terminal York-Landrace x Duroc con peso vivo inicial de $68.4 \pm 2.4 \mathrm{~kg}$. Los animales se alojaron individualmente en corrales con piso de concreto $\left(1.4 \mathrm{~m}^{2}\right)$, equipado con bebedero de acero inoxidable y comedero de plástico. Los cerdos se asignaron aleatoriamente por sexo, a cada uno de los dos tratamientos: $\mathrm{AH}$, alimentación húmeda en una proporción de 1: 1 (dieta: agua); AS, alimento seco. La dieta ofrecida a los cerdos se elaboró con base en de grano de sorgo molido, harina de soya y premezcla de vitaminas y minerales, formulada con $3200 \mathrm{kcal} \mathrm{EM} / \mathrm{kg}$ y $15 \%$ de proteína cruda, para cubrir o exceder los requerimientos nutricionales de cerdos en el rango de peso de 50 a $120 \mathrm{~kg}, \mathrm{NRC}^{(7)}$.

\section{Procedimiento experimental}

Durante el período experimental, la temperatura ambiente mínima y máxima se registró diariamente a nivel del corral, utilizando un termómetro digital (STEREN ${ }^{\circledR}$, modelo TER100, China). El periodo de adaptación a los corrales y la alimentación fue de una semana, seguida de cinco semanas de prueba. El peso vivo de los cerdos se registró semanalmente para calcular la ganancia de peso diario promedio (GDP). La alimentación ofrecida y rechazada se registró diariamente para calcular semanalmente el consumo diario de alimento $(\mathrm{CDA})$, y la relación ganancia/consumo (eficiencia alimenticia = EA).

Al final del experimento, se sacrificaron todos los cerdos en un rastro TIF (Tipo Inspección Federal). Se registró el peso de canal caliente (CC) y canal fría (CF; 24 h post-sacrificio, $\left.2{ }^{\circ} \mathrm{C}\right)$. Se midió la longitud de la canal registrando la distancia $(\mathrm{cm})$ entre la $6^{\mathrm{a}}$ vértebra 
cervical y el hueso de la cadera ${ }^{(11)}$; se registraron los pesos de los cortes primarios de la canal: pierna, paleta, lomo y costillas de acuerdo a la Norma Mexicana de Productos Pecuarios ${ }^{(14)}$.

\section{Análisis de laboratorio de la carne}

Después de 24 h post mortem, el $\mathrm{pH}$, color y la capacidad de retención de agua (CRA, \%) del músculo Longissimus dorsi (LD) se determinaron por cuadruplicado para cada muestra $^{(11)}$. En el presente estudio, el $\mathrm{pH}$ del músculo se determinó introduciendo directamente el electrodo de un potenciómetro de punción (Orion 3 star Thermo Fisher Scientific, USA).

Para evaluar la calidad de la carne, una muestra de músculo LD fue tomada de entre la $10^{\mathrm{a}} \mathrm{y}$ $12^{\mathrm{a}}$ costilla, y fue conservada a $-20^{\circ} \mathrm{C}$ hasta su análisis. El color se analizó con un colorímetro (Minolta Chroma Meter 2002, Konica Minolta Holdings, Inc., Tokyo, Japón) y los valores se expresaron con base al Sistema CIE ( $L^{*}, a^{*}$ y $\left.b^{*}\right)$. La CRA se determinó por el método de compresión anteriormente descrito ${ }^{(15)}$.

La fuerza de corte $(\mathrm{FC})$ se midió en cuatro rectángulos $(4 \times 2 \times 2 \mathrm{~cm})$ de cada muestra de $\mathrm{LD}$, en cortes realizados en paralelo a la dirección de las fibras musculares, utilizando un texturómetro (TA.XT2i Stable Micro Systems Serrey, England) equipado con una navaja Warner-Bratzler. Las condiciones de corte fueron velocidad de $2 \mathrm{mms}^{-1}$ en la prueba previa, $2 \mathrm{mms}^{-1}$ en la prueba, $10 \mathrm{mms}^{-1}$ en la prueba posterior, y a una distancia de $30 \mathrm{~mm}^{(16)}$.

El análisis de perfil de textura (APT) de las muestras de LD fue realizado con un texturómetro (TA.XT2i Stable Micro Systems Serrey, England), utilizando cuatro cubos estandarizados de $2 \mathrm{~cm}$ por cada muestra, los cuales se obtuvieron perpendiculares a la dirección de las fibras musculares. Se usó un pistón cilíndrico para comprimir la muestra al $60 \%$ de la altura original, durante dos ciclos de compresión, con un intervalo de tiempo de 5 seg entre ellos. Las curvas de deformación fuerza-tiempo se obtuvieron a partir de las condiciones establecidas de velocidad, previa a la prueba $1.0 \mathrm{mms}^{-1}$, en la prueba $5.0 \mathrm{mms}^{-1}$ y posterior a la prueba de $5.0 \mathrm{mms}^{-1}$.

La dureza (g), adhesividad (g/seg), elasticidad (mm), cohesividad, gomosidad (g), masticabilidad $(\mathrm{g} \mathrm{mm})$ y la resistencia se obtuvieron de acuerdo a reportes anteriores ${ }^{(17,18)}$. Todas las muestras de carne se analizaron para determinar el contenido de proteína por el método 990.03 de la $\mathrm{AOAC}^{(19)}$. 


\section{Análisis económico}

El ingreso del crecimiento animal se calculó considerando un precio del cerdo vivo de \$ $32.00 \mathrm{MN} / \mathrm{kg}$, multiplicado por el respectivo aumento de peso de cada animal. El costo de la alimentación se calculó considerando el precio del alimento para ambos tratamientos (\$5.90 $\mathrm{MN} / \mathrm{kg}$ ), multiplicado por el consumo respectivo de cada animal. Estas dos variables se usaron para calcular la diferencia del ingreso para el crecimiento, menos el costo de alimentación. Los precios del cerdo vivo y el costo de la alimentación se obtuvieron con los precios base de agosto-septiembre de 2018 publicados por la Confederación de Porcicultores de México ${ }^{(20)}$, y el Sistema Nacional de Información e Integración de Mercados de México $^{(21)}$.

\section{Análisis estadístico}

Los datos se analizaron bajo un diseño de bloques completos al azar, utilizando el paquete estadístico SPSS versión 22 (Versión 2013. IBM SPSS Statistics para Windows, Versión 22.0, Armonk, NY: IBM Corp.). Los datos se presentan como medias y las diferencias significativas $(P<0.05)$ se determinaron mediante la prueba de Tukey.

\section{Resultados}

La temperatura ambiente durante el experimento fluctuó desde una mínima de $9.1^{\circ} \mathrm{C}$ a una máxima de $35.3{ }^{\circ} \mathrm{C}$, con promedio de $27.3{ }^{\circ} \mathrm{C}$ durante el estudio. En el Cuadro 1 se muestran los resultados de comportamiento productivo después de cinco semanas experimentales. El peso vivo de los cerdos con alimentación húmeda fue mayor $(P<0.01)$ al final de las semanas 4 y 5 , y de todo el estudio, con respecto a cerdos que recibieron alimento seco. Las diferencias en peso vivo entre tratamientos se fueron acentuando sobre las semanas experimentales $(P<0.01)$. Aunque la GDP no fue estadísticamente diferente en cada una de las semanas, sí lo fue en forma global en los cerdos con el alimento húmedo ( $P<0.01$; Figura 1$)$. El CDA fue menor en la semana 1 y mayor en la semana 5 cuando se ofreció húmedo $(P<0.01)$, pero no fue diferente a lo largo de todo el estudio $(P>0.10)$. La variable EA $(P<0.05)$ fue mejor con el alimento húmedo en las semanas 1 y 3 (Figura 2), y en todo el estudio. 
Cuadro 1: Efecto de ofrecer alimentación húmeda (AH) o alimentación seca (AS) sobre peso vivo, CDA, GDP y EA de cerdos en finalización (68 a $108 \mathrm{~kg}$ ), por cada semana experimental

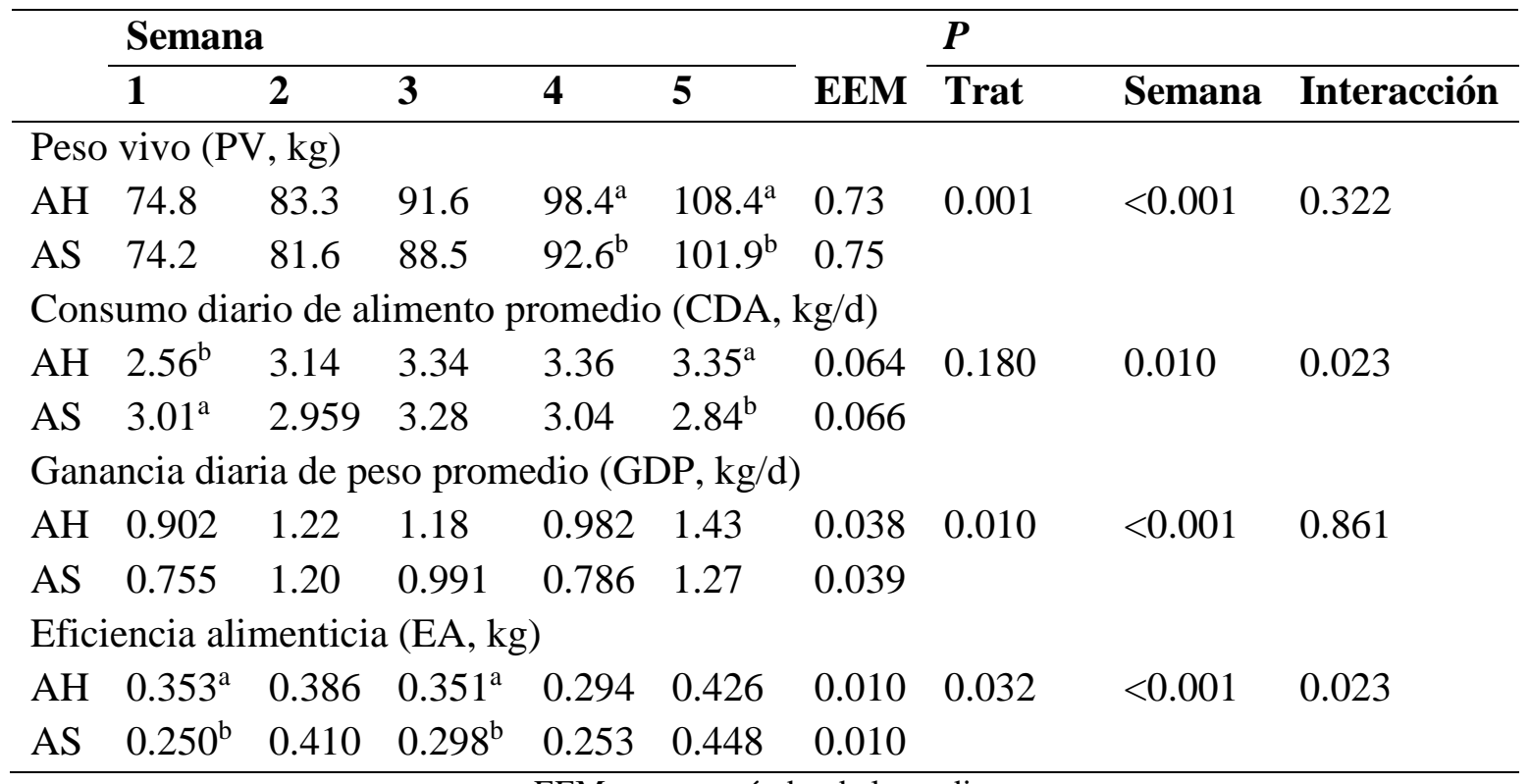
EEM= error estándar de la media.

${ }^{\mathrm{a}, \mathrm{b}}$ Medias con letras diferentes dentro de la misma columna para cada variable son diferentes $(P<0.05)$.

Figura 1: Consumo diario de alimento (Media \pm EEM) de los cerdos en fase final (68 a 108 $\mathrm{kg}$ ) alimentados con alimentación húmeda (AH) o alimentación seca (AS)

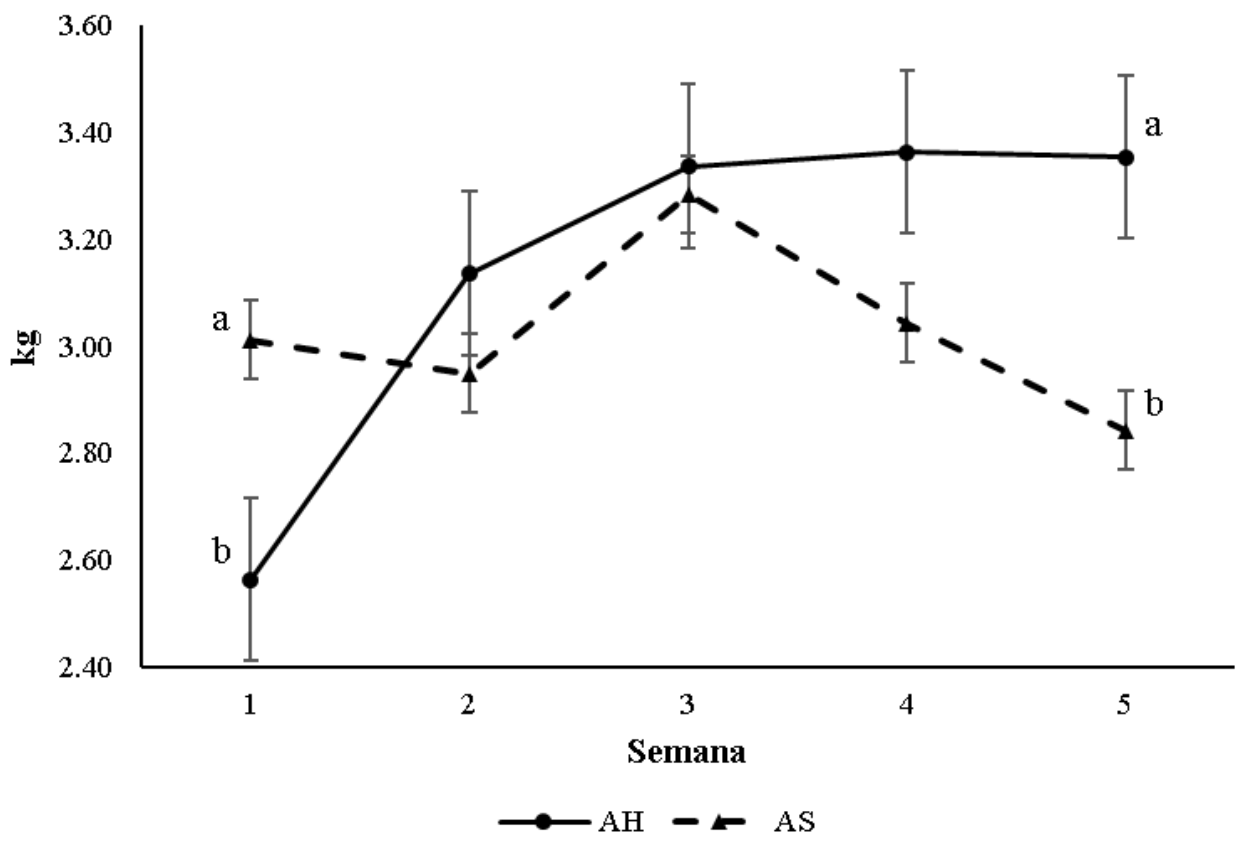

${ }^{\mathrm{a}, \mathrm{b}}$ Diferencia significativa $(P<0.05)$ entre grupos. 
Figura 2: Eficiencia alimenticia (Media \pm EEM) medidas semanalmente de los cerdos en la fase final (68 a $108 \mathrm{~kg}$ ), alimentados con alimentación húmeda (AH) o alimentación seca

(AS)

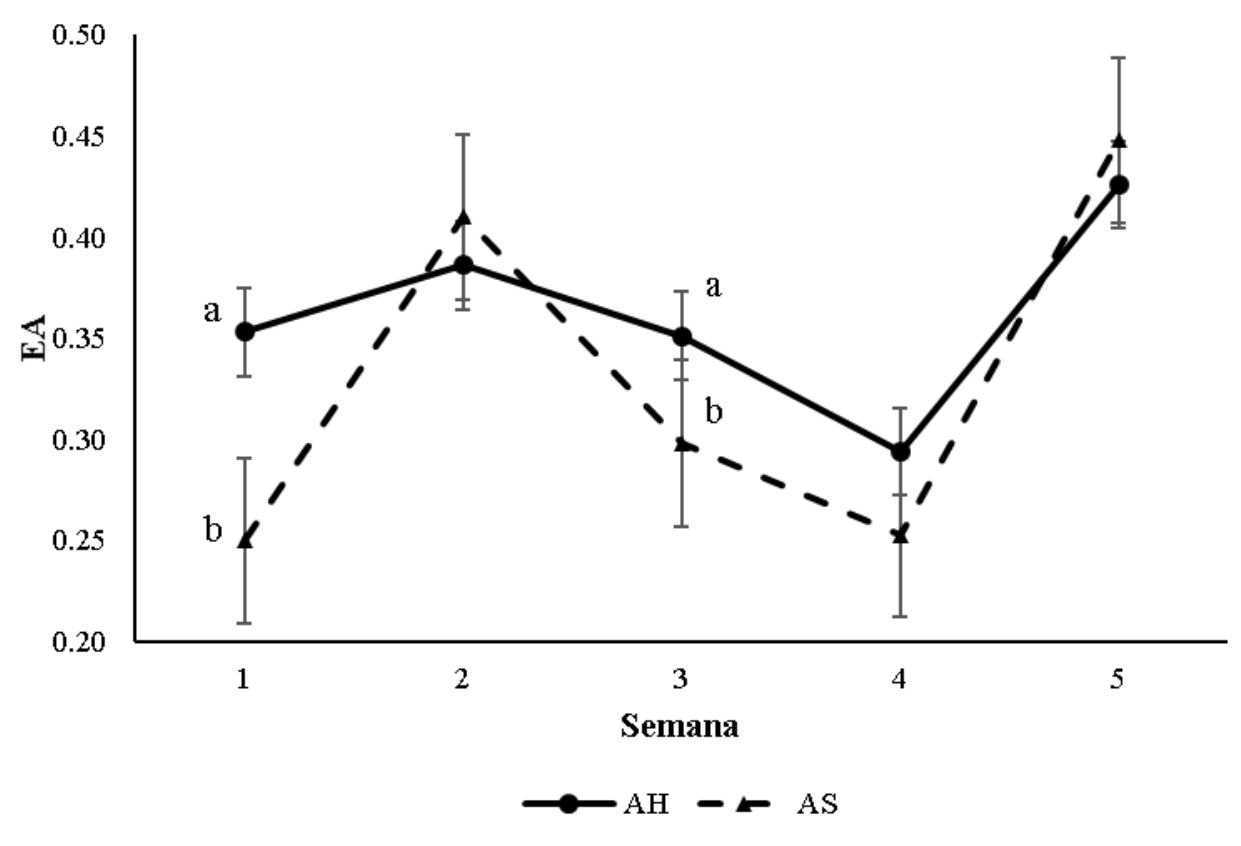

${ }^{\mathrm{a}, \mathrm{b}}$ Diferencia significativa $(P<0.05)$ entre grupos.

\section{Peso de los componentes de la canal}

Los cerdos con alimentación húmeda tuvieron mayor peso de canal caliente $(P=0.019)$ y fría $(P=0.021)$ que los cerdos que recibieron alimentación seca (Cuadro 2). La longitud de la canal no fue diferente $(P>0.05)$ para los dos tratamientos. El peso promedio de la pierna y de la piel con grasa fue mayor $(P=0.04)$ en los cerdos con alimentación húmeda, que en los cerdos de alimentación seca. Los pesos del lomo, costilla, paletas y patas no fueron diferentes entre los cerdos alimentados con una dieta húmeda o seca $(P>0.05)$. 
Cuadro 2: Características de la canal y peso de los principales componentes de la canal de cerdos sacrificados a $108 \mathrm{~kg}$ de peso vivo, con alimentación húmeda $(\mathrm{AH})$ o alimentación seca (AS)

\begin{tabular}{lllll}
\hline \multirow{2}{*}{ Concepto } & \multicolumn{4}{c}{ Tratamiento } \\
\cline { 2 - 5 } & AH & AS & EEM & $\boldsymbol{P}$ \\
\hline Medidas de la canal & & & & \\
$\quad$ Peso canal caliente, kg & 90.80 & 84.80 & 1.599 & 0.019 \\
$\quad$ Peso canal fría, kg & 89.13 & 83.39 & 1.564 & 0.021 \\
$\quad$ Longitud de la canal, cm & 81.56 & 80.94 & 0.985 & 0.661 \\
Peso promedio de las piezas, media canal, kg & & & & \\
$\quad$ Pierna & 10.33 & 9.78 & 0.172 & 0.040 \\
Lomo & 9.33 & 9.34 & 0.212 & 0.967 \\
Costilla & 5.66 & 5.03 & 0.254 & 0.097 \\
Paleta & 4.45 & 4.25 & 0.109 & 0.214 \\
Piel + grasa & 11.63 & 9.65 & 0.468 & 0.009 \\
Patas & 0.739 & 0.694 & 0.019 & 0.120 \\
\hline
\end{tabular}

$\mathrm{EEM}=$ error estándar de la media.

\section{Características fisicoquímicas y de textura de la carne}

Las características fisicoquímicas y de textura de la carne se presentan en el Cuadro 3. No se observaron diferencias en el contenido de proteína y carbono, $\mathrm{pH}$ y capacidad de retención de agua en la carne $(P>0.05)$ entre los tratamientos. La dureza, gomosidad, masticabilidad y resistencia de la carne fue mayor $(P<0.05)$ en los cerdos con alimentación seca que en los de alimentación húmeda. Las características de fuerza de corte, adhesividad, elasticidad y cohesividad no fueron diferentes $(P>0.05)$ entre los tratamientos.

Cuadro 3: Valores de las características fisicoquímicas y textura de la carne de los cerdos sacrificados a $108 \mathrm{~kg}$ de PV, con alimentación húmeda (AH) y alimentación seca (AS)

\begin{tabular}{lllll}
\hline \multirow{2}{*}{ Características } & \multicolumn{2}{c}{ Tratamiento } & \multirow{2}{*}{ EEM } & \multirow{2}{*}{$\boldsymbol{P}$} \\
\cline { 2 - 3 } & AH & AS & & \\
\hline Fisicoquímicas & & & & \\
$\quad$ Proteína, \% MS & 25.70 & 25.31 & 0.300 & 0.372 \\
$\quad$ Carbono, \% MS & 16.39 & 16.07 & 0.235 & 0.353 \\
pH & 5.50 & 5.48 & 0.018 & 0.506 \\
$\quad$ CRA, \% & 64.31 & 62.98 & 0.864 & 0.284 \\
Textura & & & & \\
$\quad$ Fuerza de corte, N & 41.22 & 37.46 & 2.384 & 0.270 \\
$\quad$ Dureza, N & 35.37 & 57.32 & 7.071 & 0.032 \\
\hline
\end{tabular}




\begin{tabular}{lllll}
\hline Adhesividad, g/seg & -25.11 & -22.66 & 1.400 & 0.216 \\
Elasticidad, mm & 0.435 & 0.457 & 0.015 & 0.324 \\
Cohesividad & 0.446 & 0.453 & 0.010 & 0.584 \\
Gomosidad, g & 15.86 & 27.16 & 3.470 & 0.025 \\
Masticabilidad, gmm & 6.30 & 11.65 & 1.367 & 0.007 \\
Resistencia & 0.237 & 0.283 & 0.013 & 0.015 \\
\hline
\end{tabular}

$\mathrm{EEM}=$ error estándar de la media; $\mathrm{CRA}=$ capacidad de retención de agua.

\section{Tendencia al color de la carne}

Los valores de luminosidad $\left(\mathrm{L}^{*}\right)$, tendencia al rojo $\left(\mathrm{a}^{*}\right)$ y amarillo $\left(\mathrm{b}^{*}\right)$, saturación $(\mathrm{C})$ y ángulo Hue $(\mathrm{H})$ de la carne no fueron diferentes $(P>0.05)$ entre los dos tratamientos (Cuadro 4).

Cuadro 4: Color de la carne medidas en músculo Longissimus dorsi de los cerdos sacrificados a $108 \mathrm{~kg}$ de peso vivo, con alimentación húmeda $(\mathrm{AH})$ o alimentación seca

(AS)

\begin{tabular}{lllll}
\hline \multirow{2}{*}{ Caracteristica } & \multicolumn{2}{l}{ Tratamiento } & EEM & \multicolumn{1}{l}{$\boldsymbol{P}$} \\
\cline { 2 - 4 } & AH & AS & 0.879 & 0.734 \\
\hline $\mathrm{L}^{*}$ & 53.62 & 54.04 & 0.221 & 0.392 \\
$\mathrm{a}^{*}$ & 17.03 & 17.3 & 0.255 & 0.656 \\
$\mathrm{~b}^{*}$ & 9.16 & 9.32 & 0.194 & 0.175 \\
$\mathrm{C}$ & 19.37 & 19.75 & 0.790 & 0.954 \\
$\mathrm{H}$ & 28.29 & 28.35 & 0.35 \\
\hline
\end{tabular}

$\mathrm{EEM}=$ error estándar de la media; $\mathrm{L}^{*}$ luminosidad; $\mathrm{a}^{*}=$ tendencia al rojo; $\mathrm{b}^{*}=$ tendencia al amarillo; $\mathrm{C}=$ saturación; H= ángulo Hue

\section{Análisis económico}

El costo de alimentación (Cuadro 5) fue similar $(P=0.180)$ para cerdos alimentados con AH que con AS (promedio $=\$ 127.61 \mathrm{MN} /$ animal durante la fase experimental). Sin embargo, debido a una mayor tasa de crecimiento, el ingreso económico fue $13 \%$ mayor $(P=0.01)$ para los cerdos que recibieron alimentación húmeda que para los cerdos alimentados con alimentación seca. La diferencia del ingreso debido al crecimiento de los cerdos menos el costo de alimentación, fue $27.3 \%$ mayor $(P=0.008)$ para los cerdos que recibieron la alimentación húmeda, comparado con la de los cerdos que recibieron alimento seco. 
Cuadro 5: Análisis de costo y utilidad económica de los cerdos en la fase final (68 a 108 $\mathrm{kg}$ ) alimentado con la dieta húmeda $(\mathrm{AH})$ o alimentación seca (AS)

\begin{tabular}{|c|c|c|c|c|}
\hline \multirow{2}{*}{ Variable económica (\$ MN) } & \multicolumn{2}{|c|}{ Tratamiento } & \multirow{2}{*}{ EEM } & \multirow{2}{*}{$\boldsymbol{P}$} \\
\hline & AH & $\mathbf{A S}$ & & \\
\hline Ingresos por el crecimiento de los cerdos & 256.00 & 226.53 & 12.145 & 0.010 \\
\hline Costo de alimentación & 130.12 & 124.96 & 3.802 & 0.180 \\
\hline $\begin{array}{l}\text { Diferencia de ingresos menos costo de } \\
\text { alimentación }\end{array}$ & 125.88 & 101.56 & 9.923 & 0.008 \\
\hline
\end{tabular}

\section{Discusión}

El presente experimento se llevó a cabo bajo condiciones climáticas representativas de muchos lugares tropicales secos, siendo este, uno de los primeros estudios realizados en la región noreste de México. Durante el experimento, los cerdos se alojaron en una nave con paredes laterales abiertas, de modo que los animales se encontraban en condiciones naturales de temperatura ambiente, que varió de 10 a $35.3{ }^{\circ} \mathrm{C}$. Estas condiciones ambientales, extremadamente variables, pudieron haber afectado la ingesta de alimento y la eficiencia alimenticia de los cerdos. Se obtuvo una interacción significativa entre semana experimental $\mathrm{x}$ tratamiento para CDA y EA, de una forma similar a lo reportado previamente ${ }^{(22,23)}$.

Se ha reportado que la exposición a temperatura ambiente de $33{ }^{\circ} \mathrm{C}$ reduce en 20 a $30 \%$ el consumo voluntario de alimento de $\operatorname{los} \operatorname{cerdos}^{(22)}$. En el presente estudio, el CDA durante la semana 1 fue $12 \%$ menor, pero en la semana 5 fue $18 \%$ mayor en los cerdos que recibieron alimentación húmeda. Aunque el consumo de alimento global no fue diferente, la tendencia a incrementar a medida que avanzó el experimento hasta llegar a ser significativamente mayor en la semana 5 indica que el humedecimiento del alimento podría ayudar a recuperar el consumo voluntario de alimento de animales bajo condiciones climáticas de estrés por calor. La alimentación húmeda incrementó también en $14 \%$ la ganancia de peso y en $8 \%$ la eficiencia alimenticia. La mayor GDP de cerdos AH, como el observado en el presente experimento, concuerda con los resultados reportados en cerdos de finalización ${ }^{(24)}$ y cerdos en crecimiento-finalización alimentados con dieta húmeda durante 90 días ${ }^{(25)}$. En conjunto, estos resultados indican que la alimentación húmeda de cerdos expuestos a condiciones de temperatura ambiente elevada podría mejorar no solamente el consumo de alimento sino también la eficiencia en la utilización del alimento. Yang et $a l^{(6)}$ reportaron una menor GDP y EA en cerdos en crecimiento-finalización alimentados con una dieta líquida con subproductos de la industria del etanol, en comparación con los cerdos alimentados con una dieta húmeda a base de maíz y harina soya. Esto sugiere que, además del tipo de alimentación (húmeda o seca), los ingredientes utilizados también son importantes en la eficiencia productiva. Posiblemente, la alimentación húmeda ofrecida a los cerdos a altas temperaturas 
ambientales, les permitió tener un mejor equilibrio de la temperatura corporal, lo que resultó en un aumento de peso y una mayor eficiencia alimenticia.

Los pesos de CC y CF mayores en los cerdos con AH registrados en el presente estudio, concuerdan con otros resultados previamente publicados ${ }^{(24)}$, en los que se reportó un mayor peso de la canal caliente, y rendimiento, aunque similar profundidad de grasa dorsal en cerdos alimentados con dieta húmeda, indicando esto la posibilidad de tener una influencia positiva de la alimentación húmeda en las características de la canal.

Se tienen reportes de similitud en grasa dorsal en cerdos con alimentación húmeda o seca ${ }^{(25)}$. Sin embargo, en pollos de engorda alimentados con una dieta húmeda tenían un mayor contenido de grasa abdominal ${ }^{(26)}$. Cuando el comedero está equipado con un suministro de agua integrado, los cerdos tienden a consumir más alimento ${ }^{(24)}$. El mayor consumo de alimento en cerdos que recibieron $\mathrm{AH}$, reportado en la semana 5 del presente trabajo, podría reflejarse en un mayor contenido de grasa dorsal.

En el presente estudio, la pierna fue más pesada en los cerdos que recibieron alimentación húmeda, que en los de alimentación seca. Esto concuerda con reportes previos ${ }^{(27)}$ en los que mayores pesos de pierna han sido registrados en cerdos con mayores tasas de crecimiento. El término calidad de la carne describe la suma de diferentes propiedades ${ }^{(28)} \mathrm{como}, \mathrm{pH}$, color, terneza, CRA y la composición química ${ }^{(29)}$, que revisten especial importancia en la evaluación sensorial ${ }^{(30)}$. En este estudio, se observaron diferencias a favor de los cerdos alimentados con una dieta húmeda en cuanto a las características de calidad y textura de la carne, tales como resistencia al corte, gomosidad, y masticabilidad ${ }^{(31)}$.

La CRA y el color son atributos importantes que determinan el atractivo visual y la terneza de la carne ${ }^{(32,33)}$. Los valores observados en este experimento, fueron similares entre tratamientos, y concuerdan con los reportados previamente ${ }^{(34,35)}$ en cerdos de finalización. En el presente trabajo, la carne de cerdos que recibieron alimento húmedo tuvo valores de dureza menores a la de cerdos que recibieron alimento seco, lo cual es indicativo de mayor terneza $^{(36)}$, que es una de las características más importantes de la calidad de la carne ${ }^{(32,37)}$. Los otros resultados del análisis del perfil de textura, tales como adhesividad y la cohesividad, que también se utilizan regularmente para determinar los atributos sensoriales ${ }^{(38)}$ no fueron diferentes entre los tratamientos en el presente trabajo.

El color es un rasgo importante de la calidad de la carne de cerdo, que puede verse afectado por diferentes factores, como la estrategia de alimentación ${ }^{(31)}$ y la raza de cerdos ${ }^{(11,12)}$. En el presente estudio, no se encontraron diferencias entre tratamientos en las variables cromáticas $\mathrm{L}^{*}, \mathrm{a}^{*}, \mathrm{~b}^{*}, \mathrm{C}$ y $\mathrm{H}$, que determinan el color. Comparado con resultados de investigaciones previas $^{(34)}$, los valores de luminosidad $\left(\mathrm{L}^{*}\right)$ en el presente trabajo para la carne de cerdos alimentados con AH y AS (53.6 y 54.0, respectivamente; Cuadro 4) fueron indicativos de 
carne "normal"(39). Valores de $\mathrm{L}^{*}$ de 58 son indicativos de carne PSE (pálido, suave, exudativo), mientras que valores inferiores a 52 indican la condición de ASD (oscuro, firme, seco $)^{(39,40,41)}$.

El análisis económico realizado en este estudio ha sido utilizado anteriormente ${ }^{(42)}$, ya que permite combinar en un solo valor (diferencia del ingreso económico menos el costo del alimento) el efecto que el tipo de alimentación evaluada tiene sobre diversas características, tales como el consumo de alimento, el aumento diario de peso y la eficiencia alimenticia. En el presente estudio, la diferencia del ingreso económico por crecimiento del cerdo, menos el costo por alimento durante cinco semanas, fue $27.3 \%$ superior para los cerdos que recibieron alimentación húmeda. El mayor porcentaje de este beneficio económico se originó en la mayor tasa de crecimiento registrada en los cerdos que recibieron alimentación húmeda. Por el contrario, no hubo diferencias en el costo de la alimentación, respecto a la forma en que se ofrecía alimento (seco o húmedo) a los cerdos. En un estudio publicado por Myers et al ${ }^{(43)}$, cerdos de entre 80 y $110 \mathrm{~kg}$ de peso alimentados utilizando un comedero que humedece el alimento tuvieron mejores datos de crecimiento que en el caso de cerdos que recibieron alimento seco. Considerando el aumento de peso y el consumo de alimento de esos cerdos ${ }^{(43)}$, al recibir alimento húmedo o seco, así como los precios del alimento y la carne de cerdo del presente experimento, el beneficio económico obtenido por Myers et al ${ }^{(43)}$ en sus cerdos, habría sido un $9.1 \%$ mejor en los que obtuvieron alimentación húmeda, que para los de alimentación seca, cifra inferior al beneficio económico registrado en el presente estudio. En resumen, con base en los resultados de este experimento, se concluye que la alimentación de cerdos en finalización con una dieta húmeda mejora las variables productivas (ganancia diaria de peso, consumo de alimento, eficiencia alimenticia), el beneficio económico, la composición de la canal y la calidad de la carne.

\section{Agradecimientos}

Los autores agradecen el soporte financiero provisto por la Convocatoria de Estancias Posdoctorales Nacionales 2018(1) del Consejo Nacional de Ciencia y Tecnología (CONACYT, México). También se agradece a la Facultad de Agronomía de la UANL por las facilidades otorgadas para la realización de la presente investigación.

\section{Conflicto de interés}

Los autores declaran no tener conflictos de interés. 


\section{Literatura citada:}

1. Chae BJ. Impacts of wet feeding of diets on growth and carcass traits in pigs. J Appl Anim Res 2000;17(1):81-96.

2. Yang JS, Lee JH, Ko TG, Kim TB, Chae BJ, Kim YY, Han InK. Effects of wet feeding of processed diets of performance, morphological change in the small intestine and nutrient digestibility in weaned pigs. Asian-Aust J Anim Sci 2001;14(9):1308-1315.

3. Moon JS, Kwon IK, Chae BJ. Effects of wet feeding of diets with or without food waste on growth performance and carcass characteristics in finishing pigs. Asian-Aust J Anim Sci 2004;17(4):504-510.

4. Brooks PH, Beal JD, Niven S. Liquid feeding of pigs: potential for reducing environmental impact and for improving productivity and food safety. Recent Adv Anim Nut Aust 2001;13:49-63.

5. Zoric M, Johansson SE, Wallgren P. Behaviour of fattening pigs fed with liquid feed and dry feed. Porcine Health Managem 2015;1:14.

6. Yang X, Nath C, Doering A, Goih J, Baidoo SK. Effects of liquid feeding of corn condensed distiller's solubles and whole stillage on growth performance, carcass characteristics, and sensory traits of pigs. J Anim Sci Biotechnol 2017;8:9.

7. NRC. National Research Council. Nutrient requirements of swine. 12th ed. Washington, DC. USA: National Academy Press; 2012.

8. Guzek D, Głąska D, Pogorzelski G, Kozań K, Pietras J, Konarska M, et al. Variation of meat quality parameters due to conformation and fat class in Limousin bulls slaughtered at 25 to 27 months of age. Asian-Aust J Anim Sci 2013;26(5):716.

9. Mir NA, Rafiq A, Kumar F, Singh V, Shukla V. Determinants of broiler chicken meat quality and factors affecting them: a review. J Food Sci Technol 2017;54(10):29973009.

10. Dalle ZA. Perception of rabbit meat quality and major factors influencing the rabbit carcass and meat quality. Livest Prod Sci 2002;75(1):11-32.

11. Choi JS, Lee HJ, Jin SK, Choi YI, Lee JJ. Comparison of carcass characteristics and meat quality between Duroc and crossbred pigs. Korean J Food Sci An 2014;34(2):238-244.

12. Chen G, Shui S, Cai Y, Na L, Su Y. Production performance, slaughtering and meat quality of different breed pigs. J Bioproces Biotech 2017;7:304. 
13. Norma Oficial Mexicana. Especificaciones técnicas para la producción, cuidado y uso de los animales de laboratorio. Ochoa MLI ed. Diario Oficial de la Federación, México (AS), México. NOM-062-ZOO-1999-2001.

14. Norma Mexicana de Productos Pecuarios. Productos pecuarios-Carne de porcino en canal-Calidad de la carne-Clasificación. Ochoa MLI ed. Diario Oficial de la Federación, México (AS), México. NMX-FF-081-2003.

15. Tsai TC, Ockerman HW. Water binding measurement of meat. J Food Sci 1981;46(3):697-701.

16. Intarapichet KO, Maikhunthod B, Thungmanee N. Physicochemical characteristics of pork fed palm oil and conjugated linoleic acid supplements. Meat Sci 2008;80:788-794.

17. Bourne MC, Kenny JF, Barnard J. Computer-assisted readout of data from texture profile analysis curves. J Texture Stud 1978;9(4):481-494.

18. Alonso V, del Mar Campo M, Provincial L, Roncalés P, Beltrán JA. Effect of protein level in commercial diets on pork meat quality. Meat Sci 2010;85(1):7-14.

19. AOAC. Official Methods of Analysis. 16th ed. Arlington, VA, USA: Association of Official Analytical Chemists. 2005.

20. Confederación de Porcicultores de México. https://www.porcimex.org/supremo.htm. Consultado 15 Ene, 2019.

21. Sistema Nacional de Información e Integración de Mercados de México http://www.economia-niim.gob.mx/nuevo/Home.aspx?opcion=Consultas/MercadosNa cionales/PreciosDeMercado/Agricolas/ConsultaGranos.aspx?SubOpcion=6|0 Consultado 15 Ene, 2019.

22. Morales A, Grageola F, García H, Arce N, Araiza B, Yáñez J, Cervantes M. Performance, serum amino acid concentrations and expression of selected genes in pair-fed growing pigs exposed to high ambient temperatures. J Anim Physiol Anim Nutr 2014;98(5):928935.

23. Cervantes M, Antoine D, Valle JA, Vásquez N, Camacho RL, Bernal H, Morales A. Effect of feed intake level on the body temperature of pigs exposed to heat stress conditions. J Thermal Biol 2018;76:1-7.

24. Bergstrom JR, Nelssen JL, Edwards LN, Tokach MD, Dritz SS, Goodband RD, DeRouchey JM. Effects of feeder design and changing source of water to a location separate from the wet-dry feeder at 4 or 8 weeks before harvest on growth, feeding behavior, and carcass characteristics of finishing pigs. J Anim Sci 2012;90(12):45674575. 
25. Chae BJ, Han InK, Kim JH, Yang CJ, Ohh SJ, Rhee YC, Chung YK. Effects of feeding processing and feeding methods on growth and carcass traits for growing-finishing pigs. Asian-Aust J Anim Sci 1997;10(2):164-169.

26. Akinola OS, Onakomaiya AO, Agunbiade JA, Oso AO. Growth performance, apparent nutrient digestibility, intestinal morphology and carcass traits of broiler chickens fed dry, wet and fermented-wet feed. Livest Sci 2015;177:103-109.

27. Lanferdini E, Andretta I, Fonseca LS, Morerira RHR, Cantarelli VS, Ferreira RA, Saraiva A, Abreu MLT. Piglet birth weight, subsequent performance, carcass traits and pork quality: A meta-analitycal study. Livest Sci 2018;214:175-179.

28. Maltin C, Balcerzak D, Tilley R, Delday M. Determinants of meat quality: tenderness. Proc Nut Soc 2003;62:337-347.

29. Kim TW, Kim CW, Yang MR, No GR, Kim SW, Kim II-S. Pork quality traits according to postmortem pH and temperature in Berkshire. Korean J Food Sci An 2016;36(1):2936.

30. Hoffman, K. 1994. What is quality? Definition, measurement and evaluation of meat quality. Meat Focus Internat 1994;3(2):73-82.

31. Rosenvold K, Andersen HJ. Factors of significance for pork quality-a review. Meat Sci 2003;64:219-237.

32. Hughes JM, Oisek SK, Purslow PP, Warner RD. A structural approach to understanding the interactions between colour, water-holding capacity and tenderness. Meat Sci 2014;98:520-532.

33. Starky CP, Gessink GH, Oddy VH, Hopkins DL. Explaining the variation in lamb longissimus shear force across and within ageing periods using protein degradation, sarcomere length and collagen characteristics. Meat Sci 2015;105:332-37.

34. Nguyen DH, Park JW, Kim IH. Effect of crumbled diet on growth performance, market day age and meat quality of growing-finishing pigs. J Appl Anim Res 2017;45(1):396399.

35. Sasaky K, Motoyama M, Narita T, Chikuni K. Effects of cooking end-point temperature and muscle part on sensory "hardness" and "chewiness" assessed scales presented in ISO11036:1994. Asian-Aust J Anim Sci 2013;26(10):1490-1495.

36. Válvoká V, Saláková A, Butchtová H, Tremlová B. Chemical, instrumental and sensory characteristics of cooked pork ham. Meat Sci 2007;77:608-615. 
37. Sørheim O, Idland J, Halvorsen EC, Frøystein T, Lea P, Hildrum KI. Influence of beef carcass stretching and chilling rate on tenderness of $m$. longissimus dorsi. Meat Sci 2001;57(1):79-85.

38. Nishinary K, Kohyama K, Kumgai H, Funami T, Bourne MC. Parameters of texture profile analysis. Food Sci Technol Res 2013;19(3):519-521.

39. Adzitey F, Nurl H. Pale soft exudative (PSE) and dark firm dry (ASD) meats: causes and measures to reduce these incidences - a mini review. Int Food Res J 2011;18:11-20.

40. Kusêc G, Kralik G, Petričević A, Gutzmirtl H, Grgurić D. Meat quality indicators and their correlation in two crosses of pigs. Agric Conspectus Scient 2003;68(2): 115-119.

41. Van Laack RLJM. Determinants of ultimate $\mathrm{pH}$ and quality of pork-NPB\#99-129. https://www.pork.org/wp-content/uploads/2007/08/99-129-VANLAACK-U-ofTN.pdf. Accesed Feb 15, 2019.

42. Guarneros-Altamirano R, Gutiérrez-Ornelas E, Bernal-Barragán H, Ávalos-Ramírez R, Castillo-Gallegos E, Olivares-Sáenz E. Acondicionamiento de becerros previos a la recría bajo pastoreo en trópico seco: Efectos sobre el peso corporal y la condición sanitaria. Rev Mex Cienc Pecu 2017;8(4):341-351.

43. Myers AJ, Goodband RD, Tokach MD, Dritz SS, DeRouchey JM, Nelssen JL. The effects of diet form and feeder design on the growth performance of finishing pigs. J Anim Sci 2013;91:3420-3428. 